\title{
Lantern to the Underworld - Following User Actions Using Bing URL Parameters When Child Exploitation Terms Are Suggested by the Search Engine
}

\author{
Nicholas G. Spear \\ NASA Office of Inspector General \\ nicholas.g.spear@nasa.gov
}

\author{
David I. Hawbecker \\ NASA Office of Inspector General \\ david.i.hawbecker@nasa.gov
}

\author{
Benjamin J. McElyea \\ NASA Office of Inspector General \\ benjamin.j.mcelyea@nasa.gov
}

\begin{abstract}
During a 2016 criminal investigation overseen by the authors, a discovery was made that the Bing search engine was suggesting search terms known to be associated with child exploitation materials. This was in response to a non-contraband search by a subject, leading to increasingly explicit suggestions. This information led to the discovery that, with a user's browser history files, the specific actions taken by a user could be isolated and tracked using Bing's own unique URL parameters. While the suggestion of contraband terms by the Bing search engine has since ceased, there has been little to no research conducted on analyzing a user's browser activity in order to determine their specific actions behind the keyboard through the use of these unique URL parameters. The purpose of this paper is to document Bing's URL parameters related to image searches (specifically the FORM parameter) in order to detail how a user's actions during searching may be determined by an analyst. The authors also provide a new tool for creating a timeline of Bing image search events when an analyst possesses a user's browser history files.
\end{abstract}

Disclaimer: Some portions of this article discuss words or language that are considered profane, vulgar, or offensive. This article is neither a legal interpretation nor a statement of [agency redacted during review] policy. Reference to any specific product or entity does not constitute an endorsement or recommendation by the [agency redacted during review]. Views and opinions expressed by the authors are those of the authors and do not necessarily reflect the view of the [agency redacted during review] or any of its officials.

\section{Introduction}

Federal law enforcement entities frequently have the unfortunate and yet undeniable necessity to investigate cases of Child Exploitation Materials (CEM) online. While many sites on the Internet are built explicitly for hosting this illegal content, often utilizing anonymization tools to cover their tracks $[1,2]$, an interesting finding was noted during a 2016 investigation in which the Bing search engine appears to have led a consumer of adult pornography down a path toward search terms related to CEM during the subject's image searches. This was done through the use of suggested searches. As is later shown, Microsoft has since resolved this issue and has done much over the years to combat online CEM $[3,4,5,6]$. However, these search suggestions are important to note for those attempting to create a detailed accounting of the choices and actions made by individuals viewing this type of material. The authors will provide resources to assist an analyst in creating a detailed timeline of the actions taken by the user when an "it wasn't me, it was the search engine" defense could possibly come into play.

\subsection{Background}

Bing currently ranks as the second-most popular search engine behind Google [7]. No academic research exists comparing the differences in usage statistics relative to explicit content, however, The Daily Dot published an article in 2014, updated in 2017, reporting on the frequented opinion that Bing is used more for explicit content than Google. A side-by-side comparison of the search engines was performed by the Daily Dot, both with SafeSearch turned off, showing much more responsive images returned by Bing than Google when searching for explicit content [8]. If true, this could explain, though not excuse, Bing's potential to provide focused paths toward very specific types of explicit material, as it would have had more data from which to learn.

The trend in criminal cases related to CEM over the last 20 years has steadily increased. A study by Wolak et al in 2012 found that arrests for technology-facilitated child sexual exploitation crimes increased by more than $300 \%$ between 2000 and 2009. [9] Additionally, the U.S Department of Justice published an interim report to the 
Attorney General providing statistics for 2010 and 2011, showing an increase of $17.6 \%$ in cases accepted for federal prosecution in just one year. A joint paper authored by Google, NCMEC, and Thorn found that reports of online child exploitation materials are growing exponentially, noting that of the $23.4 \mathrm{M}$ reports of abuse materials, 9.6M (40\%) occurred in 2017 alone. [10] The need for work in this area is an unfortunate reality and is increasingly important as these types of cases appear in federal court.

Research indicates that viewers can devolve over time in their explicit searches from terms such as "young teen sex pictures" to eventually searching less ambiguous terms meant to eliminate the false positives for more explicit and often illegal content. [11] These terms can be gathered from Thumbnail Gallery Porn (TGP) sites, which do not host content themselves, but link to external sites instead, and Steel notes that viewers will sometimes utilize search engines such as Google or Bing's Image Search as a pseudo-TGP site. [11] Indeed, our investigation found that the subject utilized Bing in exactly this way.

Steel later writes in a separate work that "[..] $]$ search engines are the most common method for finding content, including [Child Sexual Exploitation Material], on the Internet." Steel goes on to claim that "[d]espite legal defense arguments that individuals "stumble across' child pornography, there is little evidence of individuals accidentally finding child pornography when surfing the web for legal content. As such, child pornography must be actively sought out by online offenders." [12] This paper shows that this may not always be the case. The research in this paper aims to inform the community that the Bing search engine previously suggested search terms directly related to CEM, which could be used to provide evidence to the contrary that offenders must always be actively seeking this material, as well as provide a helpful method for detailing the explicit actions taken by a user. The authors discuss the methodology used to unravel the meanings behind Bing's URL parameters in hopes that, as the web changes, others will be able to utilize similar methods in their own investigations. While the focus of this paper is on the use for investigations involving CEM, its application is by no means limited to these types of investigations.

Finally, several of these parameters have been updated since the initial case study presented in section 2. As they are likely to change again, this paper is not meant to act as a long-term guide for all of the various URL parameters (and their meanings) that an analyst may encounter, but instead an explanation during a snapshot in time, as well as a helpful resource for anyone wishing to understand the processes used here at a future date should the need arise.

\subsection{Existing Research}

Little to no research has been done on tracing a user's activity and determining activities through the use of Bing URL parameters, and the authors have located no research to date which has combined this with a focus on investigating child exploitation. Browser forensics, however, is not a new topic. Resources such as [13, 14, 15] detail the vast trove of artifacts which can be extracted from a computing system.

Horsman provides recent technical notes on various searching functions of several popular browsers, however, only two of Bing's FORM parameters are described (RESTAB and QSRE)[16].

The closest available tool for parsing Bing's FORM parameters is the URL forensic tool "Unfurl" [17]. Unfurl will break down the various components of a URL and also provides generic parsers for encoded information within that URL, such as timestamps, UUIDs, and base64 data. However, when used with a Bing image search URL, Unfurl simply reports the Bing FORM parameters with no context to their meaning. Additionally, while Unfurl works well to provide a visual breakdown of the components of a single URL, Lantern is designed to not only define the FORM parameters, but also ingest the many entries located within browser history files. This gives Lantern the ability to then create a timeline of user activity, detailing each step taken by a user in order to arrive at a particular image. The authors believe this work could immediately benefit tools such as Unfurl by providing context to currently unknown URL components. In particular, Unfurl currently has at least one open issue on Github noting minimal information within the Bing search URL parser (https://github.com/obsidianforensics/unfurl/issues/88).

In 2019, the New York Times published an investigation into online child exploitation images, and found that "[b]oth recirculated and new images occupy all corners of the internet, including a range of platforms as diverse as Facebook Messenger, Microsoft's Bing search engine and the storage service Dropbox" [18].

Additionally, there has been a great deal of research done on the psychology of individuals that seek out CEM [19, 20, 21], but a good deal of these studies mostly focus on the likelihood that offenders will become contact sex offenders. There has been no research done on how the suggestions or results of a search engine may influence the user with respect to searching for pornography, or how the suggestibility of terms may influence the actions of the user. 


\subsection{Overview}

In section 2, we give a brief real-world example of the path taken through Bing search suggestions originating from a manual search for adult pornography to a search consisting of terms associated with child exploitation. In section 3, we discuss our research motivations and methodologies, providing an overview of our results. In section 4 , we discuss the tool created by the authors to automate the analysis of Bing URL results. In section 5 , we make use of the provided tool in order to create a timeline of events. In section 6, we discuss the positive efforts by Bing and Microsoft to combat online CEM. In section 7, we discuss areas of future work. In section 8 , we provide our conclusions, commenting briefly on the implications for both investigators and technology companies.

\section{Bing Suggested Searches: Real-World Example}

Around September 2016, the authors were made aware of an incident in which a subject was suspected of visiting a site identified by a popular web-filter as known Child Exploitation Material. Working backwards from the alerted URL, the analyst in charge of examination for this case was able to determine (through artifacts local to the system as well as network traffic log data) the events leading up to the subject having accessed this known contraband site. The subject had been utilizing the Internet Explorer 10 web browser on a Windows 7 operating system. Magnet Forensics Axiom was used to extract the WebCacheV01.dat browser cache files located in [ROOT] \Users \[user] \AppData \Local \Mic rosoft $\backslash$ Windows $\backslash$ WebCache $\backslash$.

The subject's actions began with a Bing image search for the term "down blouse," and by clicking through progressive suggestions made by Bing, ended at image searches for terms such as "boy girl $10 \mathrm{y} f \mathrm{fu}^{* *}$ " and "13Y webcam." The terms "10y" and "13Y" are common occurrences in cases of child exploitation - ' $y$ ' or 'yo' is meant as a shortened version of "year-old", and thus in this instance, these terms would be referring to "10-year-old" and "13-year-old". It's important to note that none of these clearly contraband search terms were ever actually typed by the subject, instead having only been selected from a list of suggestions made by the Bing search engine. In some instances, Bing made new suggestions based on the subject's clicking on a particular image. When an image is selected in the Bing image search grid, it is enlarged, and suggestions related to that specific image will appear, providing a way to fine-tune additional searches. This lack of deliberate search action by the user led the authors to attempt to recreate a specific timeline of events in order to establish a detailed account of the specific actions taken by the user, as it could be argued in court that the subject never actually performed any searches beyond the initial query for "down blouse".

A listing of terms, extracted from the Bing search URLs, which were pulled directly from the WebCache local to the subject's system, is shown below. As was shown, and is discussed later, only item \#1 was manually typed by the user. The rest of the terms were suggested to the user by Bing, requiring only for the user to click on the suggested term of interest.

1. down blouses for 2015

2. Accidental Downblouse Nipple

3. Teen School Nip Slip

4. Teen School Girls

5. Cute Girls

6. Cute Teen Girls

7. Cute Girls Nude $12 \mathrm{Y}$

8. Boy Girl 10Y Fu**

The authors noted the use of "FORM" as URL parameters in the data, and began working to attribute these IDs to specific actions taken by the subject, which resulted in the ability to generate an accurate timeline of operations actively taken by the subject, despite not having manually typed search terms. After the case was closed in 2019, the authors have worked to ensure the findings are made available to analysts who may find them useful.

\section{Motivation and Methodology}

As investigators began reviewing the web traffic located on the subject's system, the use of various URL parameters for different search strings appeared to indicate that these parameters were tied to specific actions being taken by the user. As a full understanding of these user actions was very relevant to the investigation, investigators began attempting to recreate the steps taken, documenting those steps, and each new URL parameter as it was noted.

These concepts were then used to form the basis for further research conducted by the authors to understand each of the possible URL parameters employed by Bing for image searches.

When utilizing Bing for image searching, users have numerous methods to ultimately arrive at a particular image or site, all of which appear to have their own unique URL parameters. Testing showed that there are many ways that a user can arrive at the Bing image search grid, to include clicking on suggested images or search terms made by the main Bing web search page, directly searching Bing Images using search 
terms, clicking a suggested search term based on an initial search, or selecting a suggested search term after selecting an image located during a previous search. An example is shown here in a sample URL from the realworld investigation outlined in section 2 :

hxxps : / / www [.] bing [.] com/images/se arch $? \mathrm{q}=$ Accidental+Downblouse+Nipple\& $\mathrm{F}$ $\mathrm{ORM}=\mathrm{IDMHDL}$

The authors noted that the "FORM=IDMHDL" parameter here indicates a user arrived at image results by scrolling down a previous image results page and clicking a suggested term listed in the search bar "stickied" to the top of the page. It quickly became apparent, however, that a different parameter can indicate a different action taken by the user. For example, in

hxxps : / /www [ . ] bing [ . ] com/images/sea rch? $q=$ Cute+Girls \&FORM=IRIBEP \&sid=3 [ redacted] \& format $=\operatorname{sn} r j$ son $\& j$ soncbid $=2$

FORM parameter IRIBEP indicates that the user arrived at Bing image results by having selected the Bingsuggested thumbnail of an image located at the top of the search results page, which was categorized by the Bing search engine as "People interested in 'Teen School Girls' also searched for 'Cute Girls"'.

Tests utilizing Google Chrome, Mozilla Firefox, Internet Explorer, and Safari browsers, as well as both Mac OS and Windows operating systems revealed that the Bing FORM parameters do not change with either of these variables. The authors began by manually stepping through each of the common actions a user may take during an image search, such as scrolling, clicking on images, opening thumbnails, or following links. For each action, the authors then noted the unique URL parameters generated by that step action. Each result was independently confirmed by a separate analyst, and each test was executed multiple times with various searches to ensure correctness.

A full and complete listing of the documented Bing URL parameters can be found at https://github.com/lanterntool/lanter

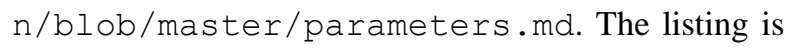
by no means meant to provide complete coverage of all possible actions a user may take; however, the authors do believe it provides a reasonable account of the most common user actions. A sample of that data is shown in Table 1 in order to provide an example of the type of translations that can be made from URL parameters to actions taken by a user.

Table 1: Example of "translated" Bing FORM Parameters

\begin{tabular}{|l|l|}
\hline $\begin{array}{l}\text { FORM }= \\
\text { RESTAB }\end{array}$ & $\begin{array}{l}\text { Indicates the user arrived at results by } \\
\text { having previously manually typed a } \\
\text { search term and then selected the Bing- } \\
\text { suggested search term } \\
\text { "[search_string]" located at the } \\
\text { top of the page. }\end{array}$ \\
\hline FORM $=$ & $\begin{array}{l}\text { Indicates the user arrived at Bing } \\
\text { image results by having selected } \\
\text { "[search_string]" in Bing Images } \\
\text { trending searches. }\end{array}$ \\
\hline FORM $=$ & $\begin{array}{l}\text { Indicates the user arrived at Bing } \\
\text { image results by having selected the } \\
\text { BNLIRS } \\
\text { "[search_string]" located in the } \\
\text { "Related Content" panel on the right } \\
\text { which appeared as a result of the user } \\
\text { having selected a thumbnail image on a } \\
\text { previous result in order to view the } \\
\text { full-size image within the browser. }\end{array}$ \\
\hline
\end{tabular}

\section{Lantern}

The most immediately applicable contribution of this paper is the Lantern tool, which can be downloaded from

https://github.com/lanterntool/lanter $\mathrm{n} /$ releases and utilized by examiners today. This tool is a single automated utility that encapsulates the manual URL analysis described in this paper by allowing examiners to load a CSV file of the URL logs retrieved from a subject's system in order to produce a translated "timeline" of events based on the interpretation of known Bing URL parameters. This can be done independent of the browser or operating system used. URL logs such as these can be exported in CSV format from common forensic tools, such as FTK, Encase, or Axiom. Results from Lantern will include a chronological timeline of the "plain language" user action events, as well as incorporate the actual user search strings into the timeline as well.

Lantern includes a full GUI, coded in Electron, a framework for building cross platform applications using JavaScript, HTML, and CSS. The full source code can be reviewed at https://github.com/lanterntool/lanter $\mathrm{n} /$, and the authors welcome additional improvements from the community.

\begin{tabular}{|l|l|} 
Parameter & Description of Meaning
\end{tabular} 


\section{Applying Bing URL Parameters to Understand User Actions}

The listing of the Bing URL parameters discussed in section 3 allows us to step through a virtual reenactment of the actions taken by a user interacting with the Bing Images search page. Utilizing the case study presented in section 2, our research can be applied by an analyst in order to make a detailed accounting of the choices made by the subject. The below narrative was created with the tool provided by the authors in order to automatically parse URL data, and represents a portion of the logs discussed in section 2 which the authors deem particularly representative of the suggestions made by Bing:

1. On 2016-09-27 at 17:34:50 UTC URL parameter QBIR indicates the user arrived at results by having manually input the search string "down blouses for 2015" on a Bing image search result page.

2. On 2016-09-27 at 17:34:57 UTC URL parameters IRPRST and thid $=$ OIP indicates the user clicked on a thumbnail image (mediaurl=[redacted $]$ ) visible in the results page of a search for "down blouses for 2015" in order to view the full-size image within the browser.

3. On 2016-09-27 at 17:35:09 UTC URL parameters IDMHDL indicates the user arrived at results by having scrolled part way down a previous image search results page until a secondary search bar appeared at the top of the page and then the user selected "Accidental Downblouse Nipple" from the Bing suggestions in this secondary search bar.

4. On 2016-09-27 at 17:35:54 URL parameter RESTAB indicates the user arrived at results by having previously typed a search term and then selected the Bing-suggested search term "Teen School Nip Slip" located at the top of the page.

5. On 2016-09-27 at 17:37:49 URL parameter IRIBEP indicates the user arrived at Bing image results by having selected the Bingsuggested thumbnail of an image located at the top of the search results page which was categorized by the Bing search engine as "People interested in [initial search] also searched for "Teen School Girls"”.

6. On 2016-09-27 at 17:38:33 URL parameter IRIBEP indicates the user arrived at Bing image results by having selected the Bingsuggested thumbnail of an image located at the top of the search results page which was categorized by the Bing search engine as "People interested in [initial search] also searched for "Cute Girls"”.

7. On 2016-09-27 at 17:38:56 URL parameter RESTAB indicates the user arrived at results by having previously manually typed a search term and then selected the Bing-suggested search term "Cute Teen Girls" located at the top of the page.

8. On 2016-09-27 at 17:55:17 UTC URL parameters IRPRST and thid $=$ OIP indicates the user clicked on a thumbnail image (mediaurl=[redacted]) visible in the results page of a search for "Cute Teen Girls" in order to view the full-size image within the browser.

9. On 2016-09-27 at 17:55:47 UTC URL parameters IDMHDL indicates the user arrived at results by having scrolled part way down a previous image search results page until a secondary search bar dropped down from the top of the page and then the user selected "Cute Girls Nude 12Y" from the Bing suggestions in this secondary search bar.

10. On 2016-09-27 at 17:56:12 UTC URL parameters IRPRST and thid=OIP indicates the user clicked on a thumbnail image (mediaurl=[redacted]) visible in the results page of a search for "Cute Girls Nude 12Y" in order to view the full-size image within the browser.

11. On 2016-09-27 at 17:56:27 UTC URL parameters IDMHDL indicates the user arrived at results by having scrolled partway down a previous image search results page until a secondary search bar dropped down from the top of the page, and then the user selected "Boy Girl 10Y Fu**" from the Bing suggestions in this secondary search bar.

These 11 steps taken by the user allowed the authors to show specific action and choices made by the subject, providing a forensic argument against the possible defense that the subject was not intentionally seeking this material out. These steps (particularly steps 9 through 11) also clearly illustrate the previous problem of Bing having been suggesting terms known to be associated with CEM.

\section{Microsoft's Efforts to Combat Online Child Exploitation Materials}

Around July 2019, which is several years after the initial incident discussed in section 2 where these findings were documented, the authors attempted in part to recreate the search suggestions noted in section 2 and 
found that Bing was no longer suggesting these terms related to contraband. Even when starting farther down the original search progression utilizing search terms such as "teen" or "young cute," Bing refused to contribute suggestions that may lead toward CEM. With Safe Search turned off, and in a sanitized browser environment, the most related search term suggestion by Bing noted during author testing was "cute," which is a common term not indicative of age, and "high school," which when followed simply led the viewer to pictures of high school buildings.

Overall, Microsoft has made significant efforts to combat online child exploitation materials. According to their online help article titled "How Bing delivers search results":

"The production and distribution of, and access to, child sexual abuse materials is universally condemned and generally illegal. ... Bing works with others in technology and industry groups, law enforcement, and governmental and non-governmental organizations to help stop the spread of this horrific content online. One way we do this is by removing pages that have been reviewed by credible agencies (or identified via Microsoft PhotoDNA) and found to contain or relate to the sexual exploitation or abuse of children.'[3]

In 2009, Microsoft partnered with a group at Dartmouth College to create PhotoDNA, a new method of image matching. This software was provided to the National Center for Missing and Exploited Children (NCMEC) [source/site] and has greatly aided in their fight against Internet Service Providers (ISPs) hosting child exploitation materials. [4]

Microsoft has also tested the use of advertisements to warn searchers that seeking child exploitation materials was illegal, encouraging the searcher to seek help. This resulted in a $67 \%$ drop in searches for child exploitation materials according to one study [5].

In 2013, Microsoft partnered with Google to help block illegal searches and remove results that are linked to Child Exploitation Materials. "It will be much harder to find that content on both Bing and Google. We are blocking content, removing content, and helping people to find the right content or also sources of help should they need that" said Nicola Hodson, Microsoft's general manager of marketing and operations. [6]

\section{Future Work}

While the authors have provided a listing of the most common Bing URL parameters for image searching, there are several next steps which proceed naturally from this work.

First, while the original nexus for the research focused solely on image searches, it was noted during this process that Bing's Web and Video searches made use of similar, and often overlapping, parameters. Extending the work to include any category of search would allow the community a broader application.

Second, these parameters are subject to change over time. Future work will create a web crawling utility to automatically document any updates to these URL parameters in the GitHub page for Lantern. Not only will this eliminate the need for manual review of URL parameters, but it will also allow for a historic documentation of URL parameters collected at various points in time, including the most current, allowing both forensic analysts and researchers to use our work to view a record of ID mappings during particular points in time, even if they have since changed.

Future work will also include updates and improvements to the Lantern tool. These updates will include being able to parse native web logs retrieved from a subject system, as opposed to needing to export the logs to a CSV, as well as automatically recognizing the date of the search queries, allowing Lantern to retrieve the most accurate URL parameters documented at that time (by the efforts of future work noted above.)

Lastly, search engines such as Google, Yahoo, or DuckDuckGo appear to utilize their own custom URL parameters for image searching, and future work will apply this same methodology to each of these search engines in turn, documenting unique components of these various URLS, and ultimately allowing Lantern to parse a wider range of URL logs collected from browser history files.

\section{Conclusions}

Through the use of a unique 2016 case study, this work has documented a real-world example of Microsoft's Bing search engine offering increasingly inappropriate suggestions, resulting in clearly contraband search terms, which had never been manually typed by the subject. This created a situation in which investigators were required to make a detailed timeline of the explicit actions taken by the user based on URL parameters alone, as it could have been argued that the user had not typed these searches, and therefore could not be held responsible for their appearance. This work led to the methods presented here, resulting in the first known large-scale documentation of the Bing URL FORM parameters. The authors also have provided a simple tool based on their results which can translate these URLs into an easily understandable timeline of events.

Ultimately this paper speaks to a larger topic of awareness and responsibility for both the IT industry and investigators when it comes to the potential repercussions of utilizing machine learning and artificial intelligence, particularly when any user online can 
provide input for training. The IT industry has a responsibility to consider how to avoid dangerous occurrences like blindly making search suggestions in their implementations and opt for inclusivity, safety, equity, and security first. Additionally, practitioners within these fields should be looking for these types of anomalies and behaviors when conducting inquiries into incidents believed to be exclusively the result of human interaction with computers, as results may not always be what they appear. Understanding this technology can help shed light on the facts of an incident when the potential means by which the person acted can be questioned.

While the issue with the suggestion of child exploitative search terms noted in this paper has been resolved by Microsoft, the authors envision other situations in which this work may be beneficial to the community both for research and practical forensic use, such as searches related to the radicalization of individuals online or the understanding of searches which may have been false positives (such as misspellings). We hope that this will assist other researchers who may encounter a situation requiring an understanding of the actions of the user behind the keyboard when the user may not have been manually typing search terms.

\section{References}

[1] C. Guitton, "A review of the available content on Tor hidden services: The case against further development.", Computers in Human Behavior 29, no. 6 (2013): 28052815.

[2] M. Spitters, V. Stefan, and M. van Staalduinen. "Towards a comprehensive insight into the thematic organization of the tor hidden services.", In 2014 IEEE Joint Intelligence and Security Informatics Conference, pp. 220-223.

[3] Microsoft. "How Bing Delivers Search Results." Bing Help. Accessed August 23, 2019. https://help.bing.microsoft.com/\#apex/18/enus $/ 10016 / 0$.

[4] Lohr, Steve. "Microsoft Tackles the Child Pornography Problem." The New York Times. The New York Times, December 16, 2009. https://bits.blogs.nytimes.com/2009/12/16/microsofttackles-the-child-pornography-problem/.

[5] T. Ly, L. Murphy, and J.P. Fedoroff. "Understanding online child sexual exploitation offenses.", Current psychiatry reports 18, no. 8 (2016): 74 .

[6] Ward, Mark. "Google and Microsoft Agree Steps to Block Abuse Images.” BBC News. BBC, November 18, 2013. https://www.bbc.com/news/uk-24980765.
[7] "Search Engine Market Share United States Of America." StatCounter Global Stats. Accessed July 27, 2019. http://gs.statcounter.com/search-engine-marketshare/all/united-states-of-america.

[8] Dickson, E J. "How Bing Became the Search Engine for Porn." The Daily Dot, March 2, 2020. https://www.dailydot.com/debug/bing-porn-searchengine/.

[9] J. Wolak, D. Finkelhor, and K. Mitchell, "Trends in law enforcement responses to technology-facilitated child sexual exploitation crimes: The Third National Juvenile Online Victimization Study (NJOV-3)", Crimes against Children Research Center, Durham, NH, 2020.

[10] E. Bursztein, E. Clarke, M. DeLaune, D.M. Elifff, N. Hsu, L. Olson, J. Shehan, M. Thakur, K. Thomas, and T. Bright, "Rethinking the detection of child sexual abuse imagery on the Internet.", In The World Wide Web Conference, 2019, pp. 2601-2607

[11] Steel, Chad MS. Digital child pornography: A practical guide for investigators. Lily Shiba Press, 2014.

[12] M.S. Steel, "Web-based child pornography: The global impact of deterrence efforts and its consumption on mobile platforms", Child Abuse \& Neglect, Volume 44, June 2015, Pages 150-158.

[13] Rathod, D., 2017. Web browser forensics: google chrome. International Journal of Advanced Research in Computer Science, 8(7), pp.896-899.

[14] Rathod, Digvijaysinh. "Mac osx forensics." International Journal of Advanced Research in Computer Engineering \& Technology (IJARCET) 6, no. 8 (2017).

[15] Said, Huwida, Noora Al Mutawa, Ibtesam Al Awadhi, and Mario Guimaraes. "Forensic analysis of private browsing artifacts." In 2011 International Conference on Innovations in Information Technology, pp. 197202. IEEE, 2011.

[16] G. Horsman, "A forensic examination of online search facility URL record structures.", Journal of forensic sciences 64, no. 1 (2019): 236-242.

[17] Benson, Ryan. "Introducing Unfurl" dfir.blog. dfir.blog, June 11, 2021. https://dfir.blog/introducing-unfurl/.

[18] Keller, Michael H., and Gabriel J. X. "The Internet Is Overrun With Images of Child Sexual Abuse. What Went Wrong?" The New York Times. The New York Times, September 29, 2019. https://www.nytimes.com/interactive/2019/09/28/us/ch ild-sex-abuse.html.

[19] Taylor, Maxwell, and Ethel Quayle. Child pornography: An internet crime. Psychology press, 2003.

[20] L. Webb, J. Craissati, and S Keen. "Characteristics of Internet child pornography offenders: A comparison with child molesters.", Sexual abuse: a journal of research and treatment 19, no. 4 (2007): 449-465.

[21] K.C. Seigfried, R.W. Lovely, and M.K. Rogers, "SelfReported Online Child Pornography Behavior: A Psychological Analysis.", International Journal of Cyber Criminology 2, no. 1, 2008. 